\title{
Screening we can believe in
}

\author{
Providers and users of chemical libraries must adopt quality and reporting standards to advance the impact of small- \\ molecule high-throughput screening.
}

$\mathrm{H}$ igh-throughput screening of chemical compound libraries to identify new biologically active small molecules is an enabling chemical biology technology with a demonstrated impact in allied fields (Nat. Chem. Biol. 3, 433, 2007). In the past decade, the greater availability of compound libraries and the growth of institutional and publically funded screening centers have improved academic access to small-molecule high-throughput screening (HTS). As a result, more scientists have applied HTS to identify chemical probes and drug leads for an expanding number of biological targets. However, with this broader adoption, the limitations and pitfalls of HTS technologies have become more apparent. To ensure that chemical library screening continues its upward trajectory, the community must establish universal standards for chemical libraries and 'best practices' for reporting the results of high-throughput chemical screening experiments.

Given that Nature Chemical Biology has been a visible forum for studies reporting HTS approaches for small-molecule discovery, we are aware of the challenges facing the community. In a special issue focusing on HTS, we published a Commentary from Inglese, Shamu and Guy, who outlined a detailed set of guidelines for reporting data from high-throughput chemical library screens (Nat. Chem. Biol. 3, 438-441, 2007). One of the points raised in this piece is echoed by two recent Nature Chemical Biology articles in which validating the structures of compound 'hits' from chemical screens presented challenges. In the first case, Nathan and colleagues reported that a compound initially identified as a screening hit failed to have activity when it was independently synthesized (Nat. Chem. Biol. 4, 609-616, 2008). In this issue, a correspondence from Eastman and colleagues (Nat. Chem. Biol. 5, 129-130, 2009) and a response from Gautier and co-workers (Nat. Chem. Biol. 5, 130,2009 ) correct the chemical structure of 'mirin', a compound identified in an earlier screen (Nat. Chem. Biol. 4, 119-125, 2008). In this case, the structure of mirin was incorrectly assigned in the original library, but the correct and misassigned structures were similar enough that standard analytical data did not readily reveal an error.

Based on our experiences with manuscripts in the HTS area and a recent survey of scientists in the HTS community, we feel that two major practical issues need immediate attention: (i) library creators and suppliers need to adopt and enforce greater quality control standards to guarantee the integrity of chemical libraries, and (ii) users of libraries need to be proactive in their HTS efforts, specifically by validating the chemical identities of their screening hits.

Library quality remains the major technical challenge facing scientists who screen chemical libraries. We urge potential screeners to 'do their homework', which includes evaluating the quality of available candidate libraries. First, they should pay attention to library composition. In particular, libraries should exclude classes of compounds that are likely to complicate chemical screening results, such as chemically unstable compounds, reactive or insoluble compounds, and so-called 'frequent hitters'. Second, users should examine the reported quality control specifications to assess the level of compound identity and purity validation.

Compound library developers and managers can lead the effort to create higher quality libraries. First, library providers should improve access to chemical library data through the development of better cheminformatic tools for users. Second, compound librarians should agree on compound exclusion criteria, perhaps using the US National Institutes of Health Molecular Libraries Small Molecule Repository compound filter as a starting point (http://mlsmr.glpg.com/MLSMR_HomePage/). Third, the community must agree on universal minimum quality control standards for characterizing the identity and purity of individual members of each library. We see the development of these standards as a community effort involving library developers, screening scientists and commercial, institutional and governmental library managers. To facilitate the process, we offer Nature Chemical Biology as a forum for discussion of the issues and final communication of the standards.

Even as library quality improves, we believe that scientists need to assume responsibility for chemical validation of the identities and purities of their screening hits. The 'gold standard' experiment demonstrates that an independently synthesized hit compound has the same chemical characterization data and biological activity as the compound identified in the screen. While this requirement may be straightforward for some screeners, other scientists may not have in-lab access to synthetic expertise. In this case, validation of the hit should be achieved through collaboration with chemists or by showing that the compound obtained from an independent source has chemical properties and biological activity identical to those of the screening hit. Screening centers and library suppliers can assist by providing compound samples in a timely manner and at a reasonable cost.

Given our commitment to ensuring that the results reported in the journal are accurate and reproducible, we will be requiring independent chemical validation of all key screening hits. Specifically, authors need to verify the identity of the compound through complete characterization data $\left({ }^{1} \mathrm{H}\right.$ NMR, ${ }^{13} \mathrm{C}$ NMR and HRMS at a minimum) and provide analytical data demonstrating compound purity. These requirements reflect the standard characterization data that we have required for all new compounds since the launch of the journal (http://www.nature.com/nchembio/authors/submit/index.html\#ch). Finally, we request that authors who submit papers to Nature Chemical Biology apply the standards outlined by Inglese, Shamu and Guy (which is freely available online) for reporting screening data in their manuscripts. We believe that adopting these screening standards is an important step for the community. We also are confident that authors and referees will apply them to enhance the rigor with which screening results are evaluated and to ensure the accuracy and completeness of the chemical screening literature. 